\title{
Research on High Frequency Radio Waves in Ship Communication
}

\author{
Xin Qiao ${ }^{\text {a) }}$ \\ North China Electric Power University, Baoding 071000, China \\ a) Corresponding author: 931621593@qq.com
}

\begin{abstract}
On high frequencies ( $\mathrm{HF}$, defined to be 3-30 MHz), radio waves can travel long distances by multiple reflections off the ionosphere and off the earth. The focus of this research is to develop an appropriate mathematical model for HF radio waves which a ship travelling across the ocean will use for communications and to receive weather and traffic reports. In order to serve a shipboard receiver moving on a turbulent ocean better, MHA model is improved with the introduction of Doppler shift, multipath delay as well as symbol error rate (SER) to indicate the communication quality in the ship's navigation.
\end{abstract}

Key words: ionosphere; mathematical; MHA mode; shipboard.

\section{FREE SPACE LOSS OF HF RADIO WAVES}

What is commonly called free space, strictly speaking, should be the vacuum while such conditions cannot be gotten in reality. In other word, the space includes the features that isotropy, zero conductivity, and relative dielectric constant and relative magnetic permeability always being one. So, the free space is an ideal position. [1]

Since the energy of the signal transmitted by the antenna is broadcast, the energy received by the receiving antenna can only be part of it. Free space loss characterizes this loss of signal during diffusion.

Set a point source antenna and put it in the free space, if the radiant power of the antenna is PT watt, which is uniformly distributed on the surface of a sphere centered on the point source antenna, and the area of spherical surface from the antenna $\mathrm{r}$ is $4 \pi \mathrm{r} 2$, so the density of power flux in the spherical surface is

$$
S=\frac{P_{T}}{4 \pi r^{2}} \times G_{T}
$$

Then calculate the input power of receiving devices. Based on the antenna theory, we can use the effective area Ae to represent the efficiency of receiving HF radio wave power by receiving antenna. Suppose that there is an antenna area, all the power of radio waves projected over this area is absorbed entirely by the load of the antenna, the area is effective area of antenna. And the effective area Ae has the following relationship with the gain coefficient of receiving antenna GR and operating wave length $\lambda$ :

$$
A_{e}=\frac{\lambda^{2}}{4 \pi} G_{R}
$$

Then the input power PR sending to the receiver is 


$$
P_{R}=S A_{e}=\frac{P_{T} G_{T}}{4 \pi r^{2}} \cdot \frac{\lambda^{2}}{4 \pi} G_{R}=\left(\frac{\lambda}{4 \pi r}\right)^{2} P_{T} G_{T} G_{R}
$$

Free space basic transmission loss Lbf is defined as the transmission loss between two ideal point source antennas in free space. It expressed the ratio of input power PT and of output power PR when the transmitting antenna gain $\mathrm{GT}=1$ as well as the receiving antenna gain $\mathrm{GR}=1$ in free space, which can also be expressed in decibels.

$$
L_{b f(d B)}=10 \lg \frac{P_{T}}{P_{R}}=20 \lg \left(\frac{4 \pi r}{\lambda}\right)
$$

\section{THE IMPROVEMENT OF MULTI-HOP ATTENUATION MODEL}

During the actual voyage for a ship, due to the influence of weather, route and other factors, a lot of complicated situations can happen in communication, the most common of which is Doppler shift and Multipath delay, both of which will cause signal attenuation and interference, thus affecting the communication of the ship.[2] Therefore, we improve the multi-hop attenuation model to allow the ship to accommodate a shipboard receiver.

\section{Simulation and Analysis}

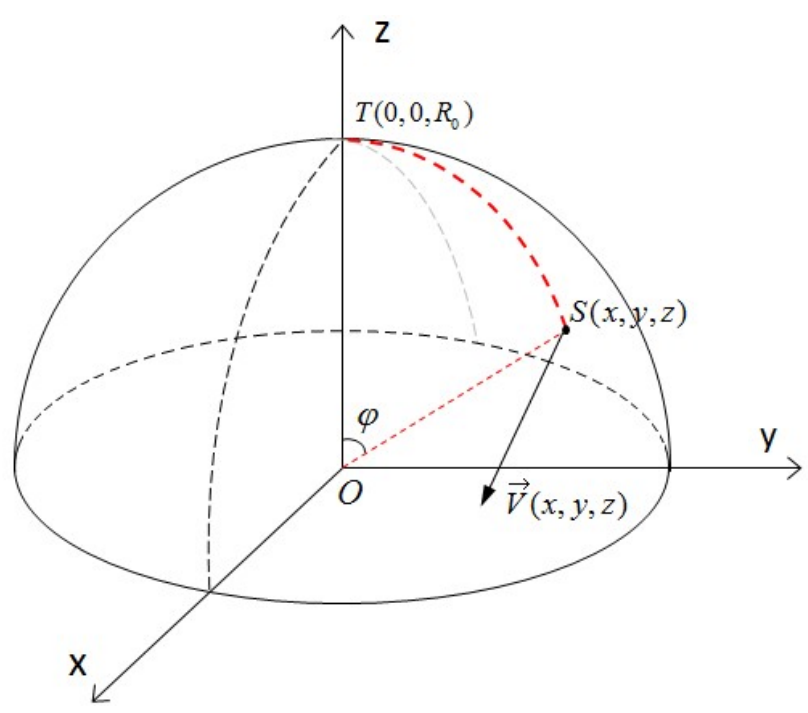

FIGURE 1. Position of source and receiver

The Doppler Effect occurs when there is relative motion between the transmitted signal and the received signal. In order to study the communication between any two points on the earth, we set up a spherical coordinate system as shown in the Fig 1 to study the relative motion between the source and the receiving source.

Assuming that the ship is sailing at any point on the surface of the earth, a signal transmitter is transmitting signal at the most north edge of the earth. It can be seen from the figure that the speed of the ship is $\mathrm{v}$, the coordinate of the launching point is $\mathrm{T}(0,0, \mathrm{R} 0)$, and the position of the ship at $\mathrm{t}$ moment is $\mathrm{S}(\mathrm{x}(\mathrm{t}), \mathrm{y}(\mathrm{t}), \mathrm{z}(\mathrm{t}))$. The angle between the navigation direction and the vertical direction is set as $\delta(t)$, the angle between the forward direction of the ship and the incident wave at $\mathrm{t}$ moment is $\gamma(\mathrm{t})$. On basis of the geometric relationship, we obtain the following relations: 


$$
\left\{\begin{array}{l}
\cos \gamma(t)=\cos \alpha(t) \cdot \cos \delta(t) \\
\cos \varphi(t)=\frac{Z(t)}{R_{0}} \\
l^{2}(t)=R_{0}^{2}+\left(H+R_{0}\right)^{2}-2 R_{0}\left(h+R_{0}\right) \cos \varphi(t) \\
\frac{l(t)}{\sin \varphi(t)}=\frac{h+R_{0}}{\sin \left(\alpha(t)+\frac{\pi}{2}\right)}
\end{array}\right.
$$

By analyzing the angle between the ship's forward direction and the incident wave at $\mathrm{t}$ moment is $\gamma(\mathrm{t})$, and then the Doppler shift fd of the ship is solved.

$$
f_{d}=f \cdot \frac{v}{c} \cdot \cos \gamma(t)
$$

\section{Multipath Delay}

Multipath effect widely exists in wireless communication technology. Due to the complexity of the environment the communication occurs, the signal may be reflected, refracted and scattered in the process of arriving at the receiving end, and it will pass through different paths to the receiver, as shown in Fig 2.

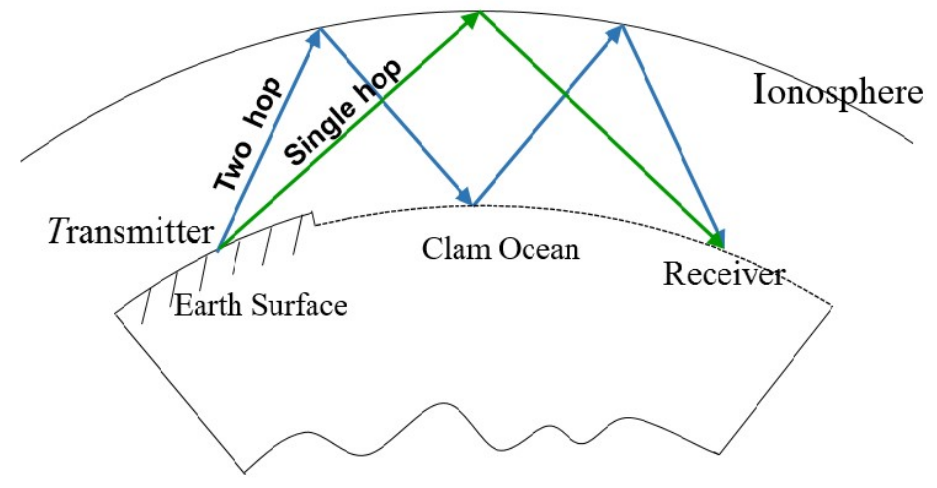

FIGURE 2. Multi-hop reflection schematic

The signal received at the receiving end is the vector sum of the signals transmitted by multiple paths. Since the attenuation and delay of each transmission path vary with time, the amplitude of the received signal varies dramatically with time due to the superposition of multiple signals with different phases, resulting in the distortion of the received signal. [3] Then, it forms the fading of the received signal which will affect the quality of the information transmission when the fading is serious.

The expression of the signal transmitted by the signal generator is

$$
s(t)=\operatorname{Re}\left[s_{l}(t) \cdot e^{j 2 \pi / f_{c} t}\right]
$$

Where, $\mathrm{sl}(\mathrm{t})$ represents the envelope of signal $\mathrm{s}(\mathrm{t})$. Because of the existence of multiple transmission paths, and the transmission delay and attenuation factors of each path vary with time. The receiving signal is expressed as

$$
x(t)=\sum_{n} a_{n}(t) \cdot s\left(t-\tau_{n}(t)\right)
$$


an $(\mathrm{t})$ is the attenuation factor corresponding to each signal while $\tau \mathrm{n}(\mathrm{t})$ is the transmission delay of each signal

\section{Calculation and Analysis}

After the improvement of the model, in order to obtain the communication time that the ship can maintain under different conditions, we introduce the symbol error rate (SER) to represent the communication quality in the course of the ship's navigation. And we use the Simulink module of MATLAB to simulate of the HF wireless communication for the signal, then to determine the change rule of the ship's communication error rate with time.

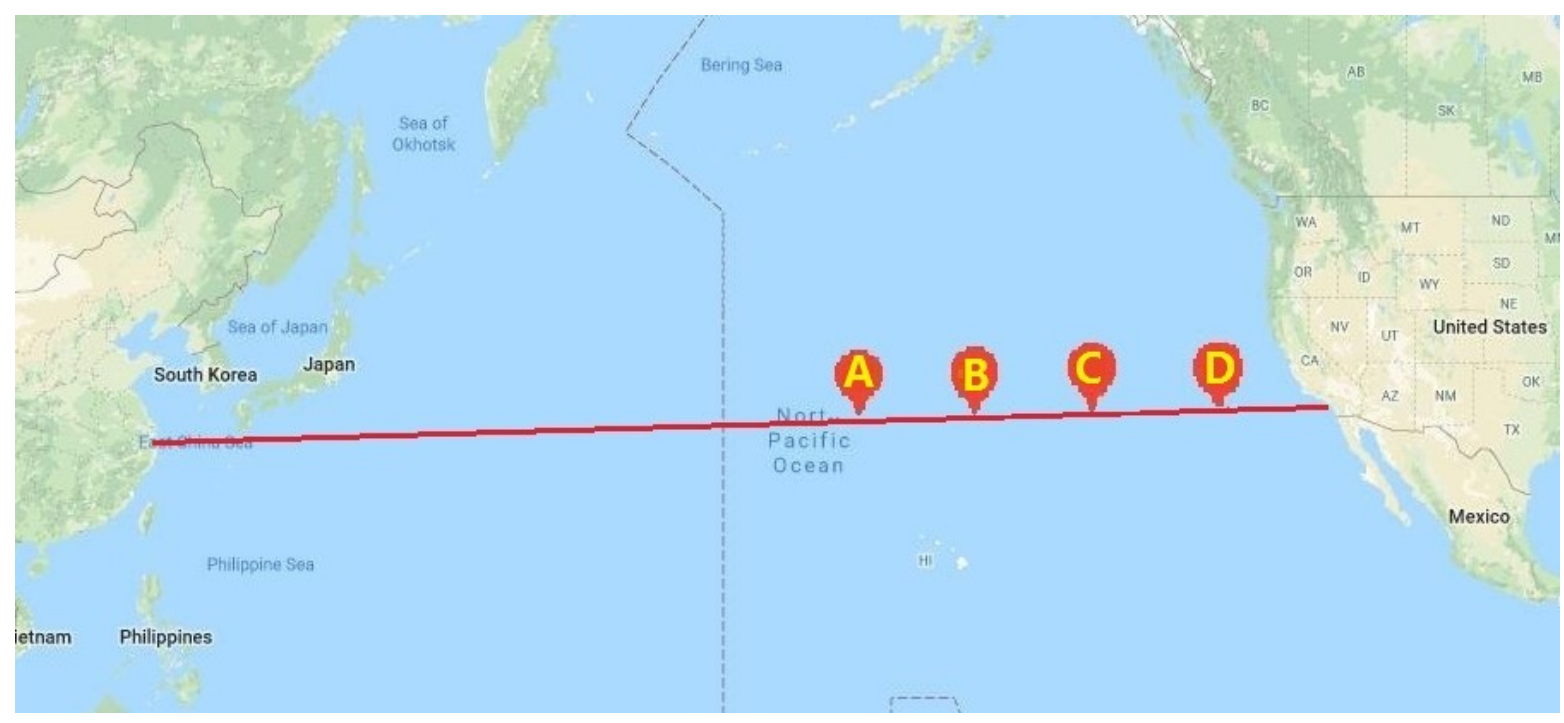

FIGURE 3. Schematic diagram of the shipping line

TABLE 1. Four positions of the ship

\begin{tabular}{ccccc}
\hline Position & $\mathrm{A}$ & $\mathrm{B}$ & $\mathrm{C}$ & $\mathrm{D}$ \\
\hline Latitude & $33.506^{\circ} \mathrm{N}$ & $33.6653^{\circ} \mathrm{N}$ & $33.474^{\circ} \mathrm{N}$ & $32.771^{\circ} \mathrm{N}$ \\
Longitude & $130.771^{\circ} \mathrm{W}$ & $143.779^{\circ} \mathrm{W}$ & $153.975^{\circ} \mathrm{W}$ & $165.752^{\circ} \mathrm{W}$ \\
\hline
\end{tabular}

To better study the reliability of our model to the actual situation, we simulate a ship sailing from Los Angeles $\left(34^{\circ} 05^{\prime} \mathrm{N}, 118^{\circ} 22^{\prime} \mathrm{W}\right)$ across the Pacific Ocean to Shanghai $\left(31^{\circ} 14^{\prime} \mathrm{N}, 121^{\circ} 27^{\prime} \mathrm{E}\right)$ with the navigation path shown in Fig 3, and there's a signal generator in Los Angeles to send weather and traffic reports to ships.

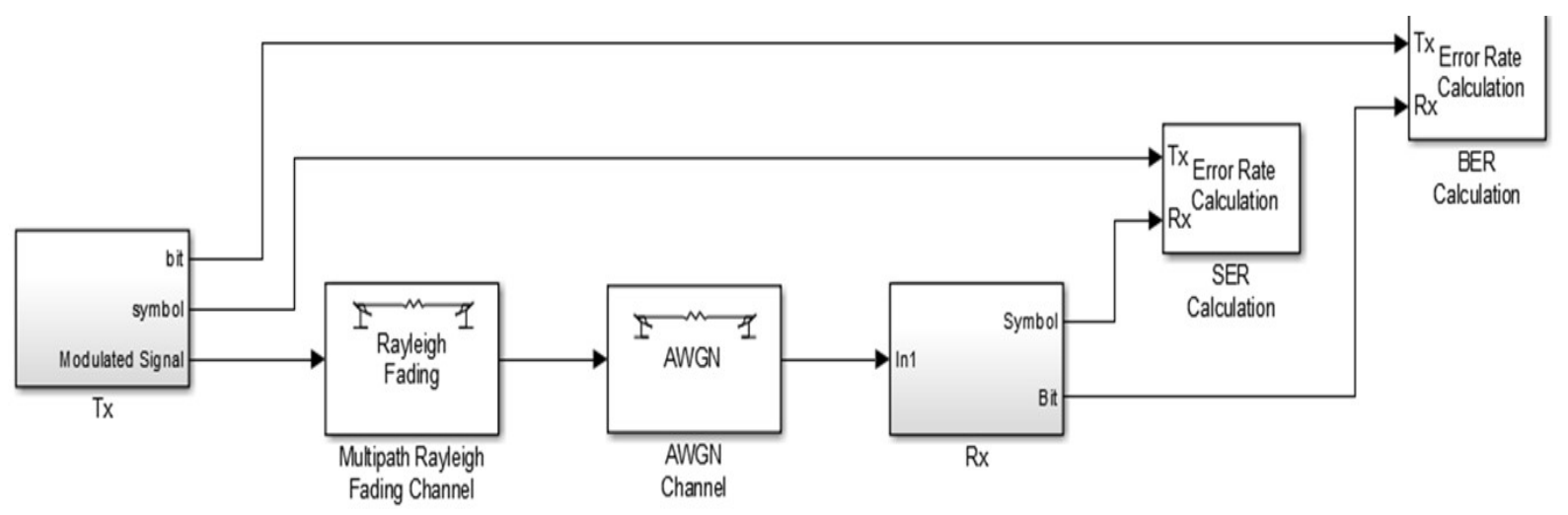

FIGURE 4. Multipath Rayleigh channel simulation module 
First of all, we built a communication module, as shown in Fig 4, in which the Multipath Reyleigh Fading is the core of the module. Each path is affected by delay time $\tau$, Doppler frequency offset fd, and gain Lb, Combining the improved MHA model with this module, two figures are obtained, which are the trend of Doppler frequency shift and multipath delay with the days of ship sailing.
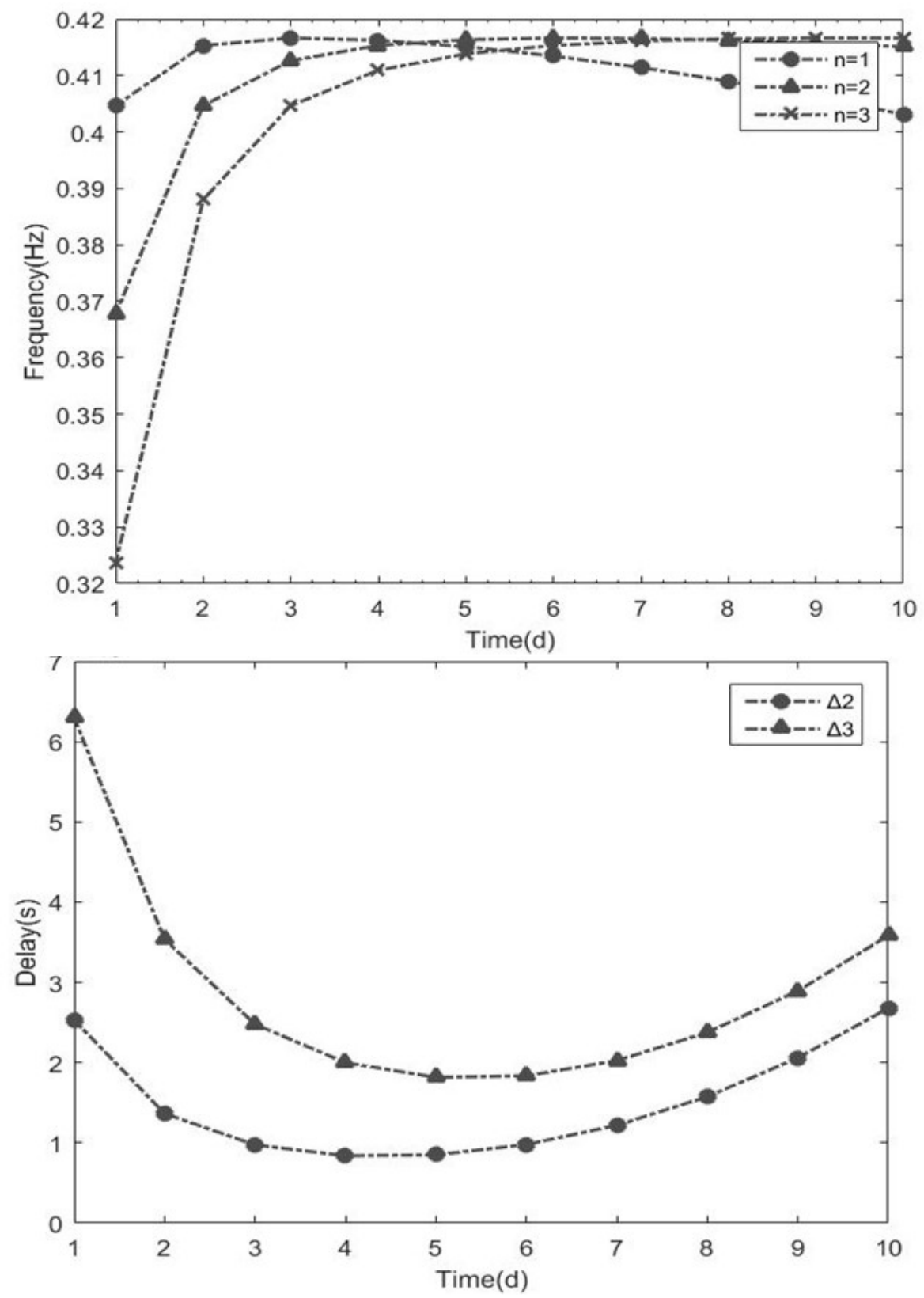

FIGURE 5. Simulink analysis

From the analysis of the two figures above, we can get the conclusions:

The Doppler frequency shift of the ship will increase with the additional number of sailing days, especially the signal whose signal reaches the ship by one hop. The multipath delay of the signal received by the vessel tends to decrease first and then tends to be gentle with the increase of the number of days. 
The main reason is that as the distance from the starting point to the vessel increases, the different paths reach the receiving point through multiple times Reflection, the physical process is very complicated.

Afterward, four locations are selected in the navigation path and to analyze the error rate of the received signals. We get the conclusions as follows:

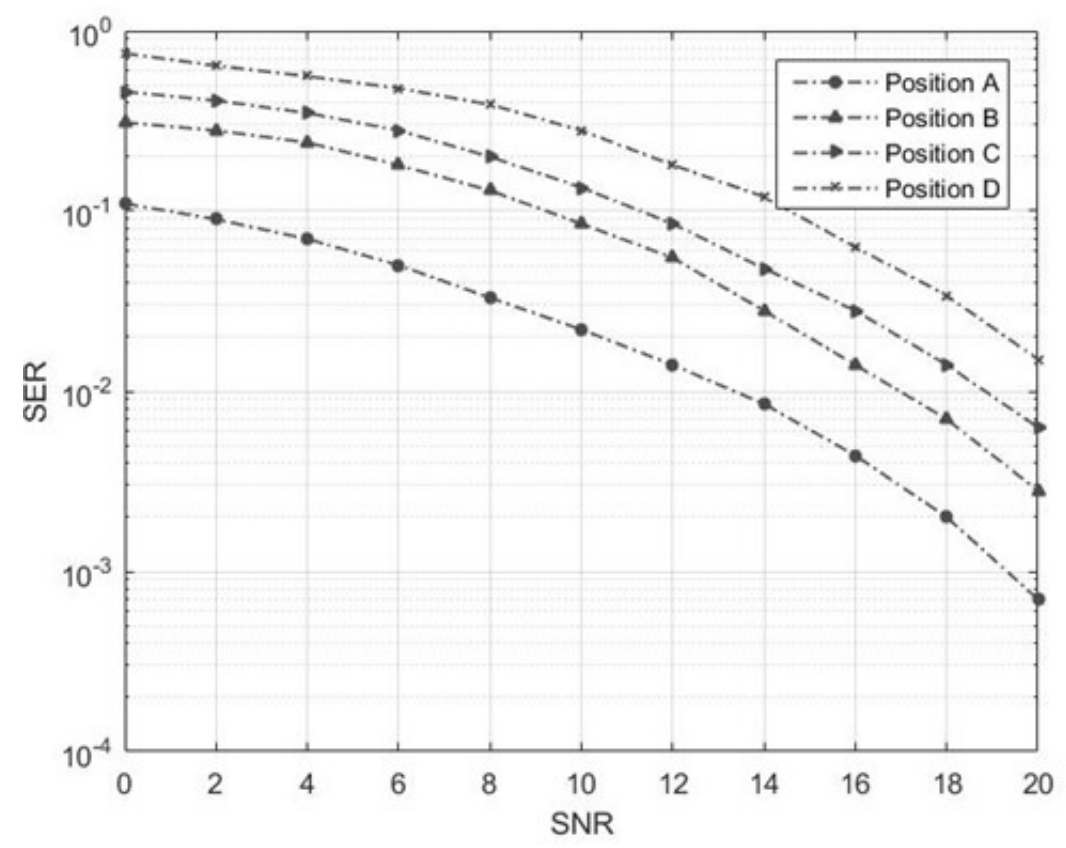

FIGURE 6. Multipath Rayleigh channel simulation module

No matter what position of the ship in the navigation, the bit error rate will decrease with the increase of signalnoise ratio, which means that the smaller the noise, the less the influence on the communication quality and the longer the ship keeps in communication.

In the process of the sailing, the ship has the farther distance from the signal transmitter, the greater the signalnoise ratio of the signal and the worse the communication quality, which results in the shortening of the communication time.

In order to ensure the communication quality, when the SNR is greater than $10 \mathrm{~dB}$, the SER should be controlled below 0.1 , so the ship in position $\mathrm{C}$ and $\mathrm{D}$ has been unable to communicate normally. Therefore, we conclude that the ship can sail for up to 4 or 5 days.

\section{REFERENCES}

1. "Comparison between the measurement and prediction of HF radio signals propagating along the mid-latitude trough $[\mathrm{J}] "<\mathrm{em}>$ The Institution of Electrical Engineers. Printed and published by The IEE Michael Faraday House Six Hills Way Stevenage SGi 2AY</em $>2003$.

2. Z. Lan, W. Xiongbin, L. Jianfei, L. Zhengyong and Y. Songhua, "A study on skywave-surface wave hybrid networking technique for HF oceanography radar," OCEANS 2014 - TAIPEI, Taipei, 2014, pp. 1-4.

3. S. Gilkhun Tanye Cemil B. Erol "Comparison of the current methods for coverage area prediction for communication in the HF band" in IEEE pp. 1888-1891 1998. 